\title{
Epidemiologia e fatores de risco relacionado a neoplasias pancreáticas: uma revisão da literatura
}

\author{
Epidemiology and risk factors related to pancreatic neoplasms: a literature review \\ Epidemiología y factores de riesgo relacionados con las neoplasias pancreáticas: revisión \\ de la literatura
}

David Martins da Silva Mello ${ }^{1 *}$, Victoria Haydée Deusdedith Neves ${ }^{1}$, Manuela Azevedo Vieira1 ${ }^{1}$ Evlyn Karolayne Bispo Andrade ${ }^{1}$, Thayná Brunelly Dórea Martins ${ }^{1}$, Ingrid Cristiane Pereira Gomes ${ }^{1}$.

\section{RESUMO}

Objetivo: Este artigo buscou analisar o perfil epidemiológico e os fatores de risco mais relevantes relacionados às neoplasias pancreáticas. Métodos: Trata-se de uma revisão da literatura atual, na qual foram incluídos 28 artigos da plataforma PUBMED entre janeiro/2016 e fevereiro/2021, utilizando os seguintes descritores: neoplasia pancreática, fatores de risco e epidemiologia. Resultados: As neoplasias pancreáticas são extremamente fatais, apresentando a terceira maior taxa de mortalidade dentre as neoplasias globalmente. Elas geralmente apresentam clínica inespecífica e tardia, com terapêutica limitada a depender do estadiamento ao diagnóstico. Os fatores de risco mais prevalentes relacionados ao câncer pancreático são a presença de pancreatite crônica, tabagismo e história prévia familiar de melanoma ou neoplasia pancreática, todavia, a dieta e alguns genes vêm demonstrando papel importante na incidência dessa patologia. Considerações finais: Deste modo, é essencial reconhecer os fatores de risco para o câncer pancreático, a fim de preveni-lo, ou ao menos, diagnosticá-lo mais precocemente para melhor resposta terapêutica.

Palavras-chave: Neoplasia pancreática, Fatores de risco, Epidemiologia.

\begin{abstract}
Objective: This article sought to analyze the epidemiological profile and the most relevant risk factors related to pancreatic neoplasms. Methods: This is a review of the current literature, in which 28 articles from the PUBMED platform were included between January / 2016 and February / 2021, using the following descriptors: pancreatic neoplasm, risk factors and epidemiology. Results: Pancreatic neoplasms are extremely fatal, with the third highest mortality rate among neoplasms globally. They usually have a nonspecific and late clinic, with limited therapy depending on the stage at diagnosis. The most prevalent risk factors related to pancreatic cancer are the presence of chronic pancreatitis, smoking and a previous family history of melanoma or pancreatic cancer, however, diet and some genes have been showing an important role in the incidence of this pathology. Final Considerations: Thus, it is essential to recognize the risk factors for pancreatic cancer to prevent it, or at least, diagnose it earlier for a better therapeutic response.
\end{abstract}

Key words: Pancreatic neoplasm, Risk factors, Epidemiology.

\footnotetext{
1 Universidade Tiradentes (UNIT), Aracaju - SE.

*E-mail: davidmsm1@gmail.com
}

SUBMETIDO EM: 4/2021

ACEITO EM: 4/2021

PUBLICADO EM: 5/2021 


\section{RESUMEN}

Objetivo: Este artículo buscó analizar el perfil epidemiológico y los factores de riesgo más relevantes relacionados con las neoplasias pancreáticas. Métodos: Se trata de una revisión de la literatura actual, en la que se incluyeron 28 artículos de la plataforma PUBMED entre enero / 2016 y febrero / 2021, utilizando los siguientes descriptores: neoplasia pancreática, factores de riesgo y epidemiología. Resultados: Las neoplasias pancreáticas son extremadamente mortales, con la tercera tasa de mortalidad más alta entre las neoplasias a nivel mundial. Suelen tener una clínica tardía e inespecífica, con terapia limitada según el estadio al momento del diagnóstico. Los factores de riesgo más prevalentes relacionados con el cáncer de páncreas son la presencia de pancreatitis crónica, tabaquismo y antecedentes familiares previos de melanoma o cáncer de páncreas, sin embargo, la dieta y algunos genes han venido mostrando un papel importante en la incidencia de esta patología. Consideraciones finales: Así, es fundamental reconocer los factores de riesgo del cáncer de páncreas para prevénirlo, o al menos diagnosticarlo antes para una mejor respuesta terapéutica.

Palabras clave: Neoplasia pancreáticas, Factores de riesgo, Epidemiología.

\section{INTRODUÇÃO}

As neoplasias pancreáticas são extremamente fatais, apresentando a terceira maior taxa de mortalidade dentre as neoplasias globalmente. Embora apresentem baixa incidência, são agressivas e possuem menos de $10 \%$ de sobrevida em cinco anos após diagnóstico (MIZRAHI J, et al., 2020). A ressecção cirúrgica é a única atual modalidade de tratamento com potencial resolutivo, mas é infrequente, devido à localização do tumor e detecção tardia. Metástase rápida e resistência à quimioterapia contribuem para um mau prognóstico (PEREIRA S, et al., 2020).

A maioria dos cânceres pancreáticos são caracterizados como adenocarcinoma ductal, enquanto uma minoria são tumores neuroendócrinos. A clínica habitual é inespecífica, normalmente tardia e o exame físico raramente apresenta alterações. Os sintomas variam de náusea, desconforto ou dor epigástrica até obstrução gástrica, de vesícula biliar, anorexia, diabetes mellitus, perda de peso ou trombose venosa (MIZRAHI J, et al., 2020). Na literatura, existem conflitos acerca dos fatores que podem levar ao desenvolvimento dessa neoplasia. Fatores ambientais incluindo tabagismo e uso de álcool, exposição a agentes químicos, alimentação e comorbidades, tais como diabetes mellitus, obesidade, síndrome metabólica e pancreatite crônica, foram postulados como pivôs no desenvolvimento do câncer pancreático (MIZRAHI J, et al., 2020).

No que tange aos fatores genéticos, aponta-se a influência do histórico familiar positivo para neoplasia pancreática ou para determinados tipos de neoplasias que predispõem o desenvolvimento de câncer pancreático, a exemplo do melanoma, bem como síndromes genéticas, como a Síndrome de Peutz-Jeghers (PEREIRA S, et al., 2020). Na medicina moderna, a identificação de fatores genéticos e ambientais que afetam o risco de desenvolvimento de câncer pancreático pode ajudar na prevenção e ampliar o espectro de vigilância em indivíduos suscetíveis, visto que a possibilidade de uma triagem custo-efetiva ainda é uma realidade distante (PEREIRA S, et al., 2020).

Esta revisão bibliográfica justifica-se pelo fato de as neoplasias pancreáticas constituírem um importante problema de saúde pública, pois apresentam uma elevada taxa de mortalidade, devido à inexistência de uma triagem eficaz, aliada ao conhecimento limitado acerca dos fatores de risco, o que contribui para um diagnóstico tardio. $\mathrm{O}$ objetivo deste estudo foi identificar as características epidemiológicas inerentes às neoplasias pancreáticas, analisando os principais fatores de risco associados.

\section{MÉTODOS}

O estudo é uma revisão integrativa da literatura com análise e síntese dos resultados observados. As questões norteadoras do artigo foram "Qual o perfil epidemiológico das neoplasias de pâncreas e quais são os principais fatores de risco associados a essa patologia?" 
O levantamento foi realizado por acesso online aos artigos utilizados nos meses de janeiro e fevereiro de 2021 com posterior análise do material coletado. Utilizando a plataforma Medical Literature Analysis and Retrieval System Online (MEDLINE), por meio do mecanismo de busca Public/Publisher MEDLINE (PubMed). Para tanto, utilizou-se a ferramenta Medical Subject Headings Terms (MeSH Terms) com os descritores a seguir: neoplasia pancreática, fatores de risco e epidemiologia. Esses descritores foram selecionados com finalidade de contemplar as questões propostas.

Os critérios de inclusão utilizados foram artigos na base de dados selecionada em português, inglês ou espanhol, publicados entre janeiro/2016 e fevereiro/2021. Foram excluídos artigos com texto incompleto e revisões literárias (Figura 1).

Figura 1 - Fluxograma de critérios de inclusão e exclusão da pesquisa.

Artigos encontrados na base de dados PubMed $(n=115)$

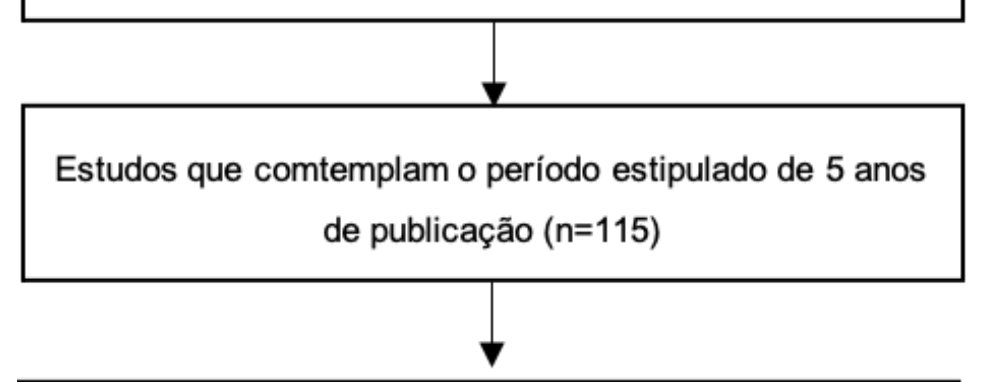

Estudos publicados em idioma português, inglês e/ou espanhol $(n=112)$

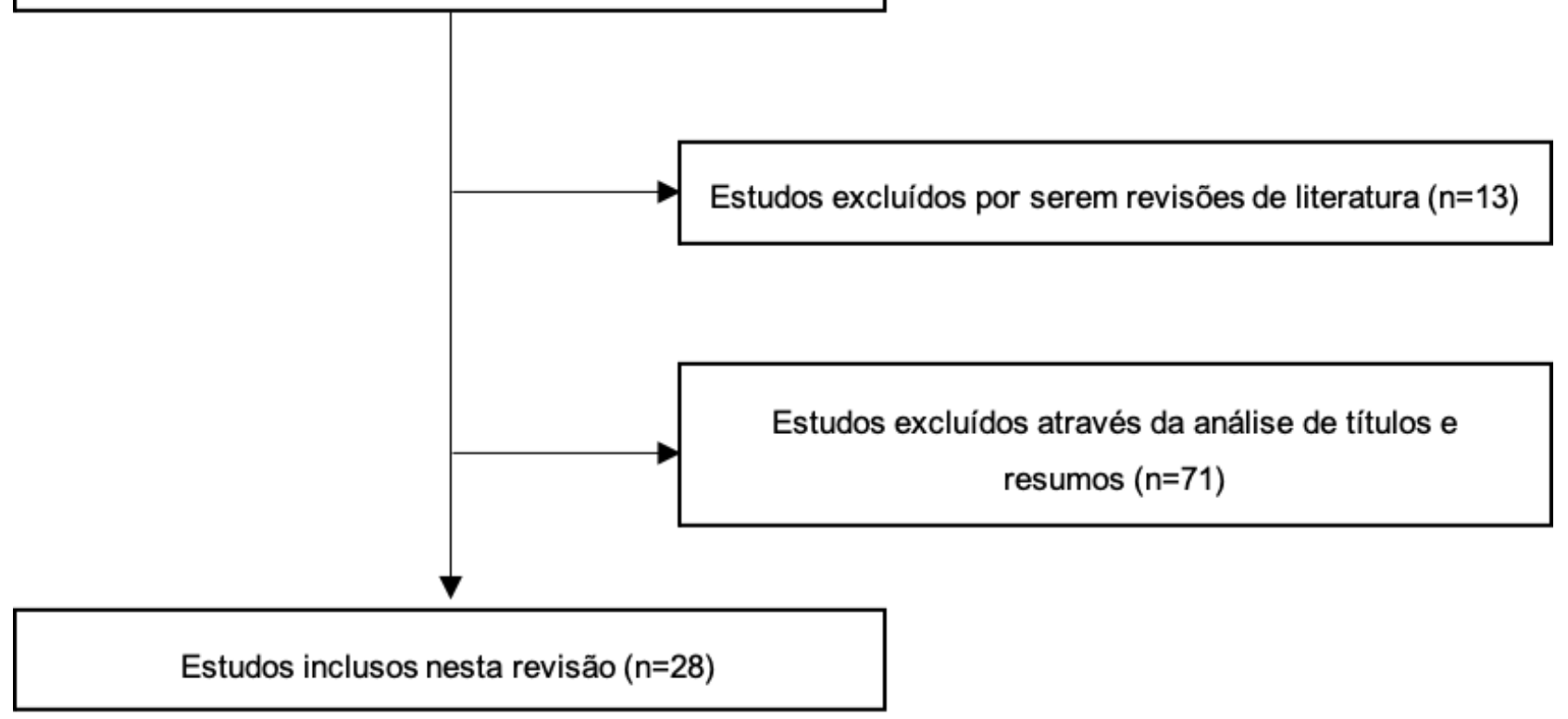

Fonte: Mello DMS, et al., 2021.

\section{RESULTADOS E DISCUSSÃO}

Foram encontrados 115 artigos na base PUBMED, dos quais 87 artigos foram excluídos por não atenderem aos critérios dispostos previamente citados, sendo incluídos ao final um total de 28 estudos (Tabela 1). 
Tabela 1 - Síntese dos principais artigos e achados sobre câncer pancreático.

\begin{tabular}{|c|c|c|}
\hline $\mathbf{N}$ & Autores (Ano) & Principais achados \\
\hline 1 & $\begin{array}{l}\text { Abdelrehim MG, et } \\
\quad \text { al. (2018) }\end{array}$ & $\begin{array}{l}\text { Corrobora a associação entre fatores da dieta e desenvolvimento de câncer } \\
\text { pancreático }(\mathrm{CP}) \text {. Maior aporte calórico está associado com aumento do risco de } \\
\mathrm{CP} \text {, enquanto consumo de vegetais é um fator de proteção. }\end{array}$ \\
\hline 2 & Ben Q, et al. (2016) & $\begin{array}{l}\text { O estudo identificou que história de CP em primeiro grau, tabagismo e diabetes } \\
\text { são fatores de risco para tumor neuroendócrino de pâncreas (PNET) não funcional. }\end{array}$ \\
\hline 3 & Boursi B, et al. (2021) & $\begin{array}{l}\text { Uso de digoxina está associado com aumento modesto do risco de CP, porém não } \\
\text { afeta sobrevida. }\end{array}$ \\
\hline 4 & $\begin{array}{l}\text { Chittiboyina S, et al. } \\
\text { (2018) }\end{array}$ & $\begin{array}{l}\text { Não houve diferença significativa entre desenvolvimento de CP e níveis de folato. } \\
\text { Foi identificado que algumas enzimas podem ter relação com risco de CP. }\end{array}$ \\
\hline 5 & $\begin{array}{l}\text { Gordon-dseagu VLZ, } \\
\text { et al. (2017) }\end{array}$ & $\begin{array}{l}\text { O estudo evidenciou que a dieta durante a adolescência e meia vida está } \\
\text { relacionada com o risco de desenvolver CP. }\end{array}$ \\
\hline 6 & Hao L, et al. (2017) & $\begin{array}{l}\text { O risco de CP é maior na população com pancreatite crônica que na população } \\
\text { geral, especialmente em idosos com histórico de tabagismo. }\end{array}$ \\
\hline 7 & $\begin{array}{l}\text { Huang BZ, et } \\
\text { al. (2019) }\end{array}$ & $\begin{array}{l}\text { Foi evidenciado que existem diferenças entre o desenvolvimento de CP dentre as } \\
\text { diferentes etnias, entretanto não foi possível estabelecer resultados definitivos. }\end{array}$ \\
\hline 8 & Jiao L, et al. (2018) & $\begin{array}{l}\text { Intervenção com uma dieta baixa em gorduras está relacionada com redução do } \\
\text { risco de desenvolver CP em mulheres com sobrepeso ou obesas. }\end{array}$ \\
\hline 9 & $\begin{array}{l}\text { Kamath GR, et al. } \\
(2019)\end{array}$ & $\begin{array}{l}\text { História prévia de câncer tem maior risco para desenvolver PNET que a população } \\
\text { geral. }\end{array}$ \\
\hline 10 & $\begin{array}{l}\text { Koyanagi VN, et al. } \\
(2018)\end{array}$ & $\begin{array}{l}\text { O estudo revelou risco fortemente positivo entre obesidade e risco de CP em } \\
\text { homens. }\end{array}$ \\
\hline 11 & $\begin{array}{l}\text { Koyanagi VN, et al. } \\
\text { (2019) }\end{array}$ & $\begin{array}{l}\text { O estudo confirma a associação entre tabagismo e risco de desenvolvimento de } \\
\text { CP. }\end{array}$ \\
\hline 12 & Luo G, et al. (2019) & $\begin{array}{l}\text { Maior consumo de carne vermelha, processados e consumo inadequado de } \\
\text { frutas/vegetais tem associação com maior risco de CP. }\end{array}$ \\
\hline 13 & Luu HN, et al. (2019) & $\begin{array}{l}\text { O estudo demonstrou que telômeros mais longos estão associados com aumento } \\
\text { de risco geral de CP. }\end{array}$ \\
\hline 14 & $\begin{array}{r}\text { Moossav } \\
(20 \\
\end{array}$ & $\begin{array}{l}\text { Os resultados mostraram associação positiva entre consumo de ópio e } \\
\text { desenvolvimento de CP. }\end{array}$ \\
\hline 15 & $\begin{array}{l}\text { Nauck MA, et al. } \\
(2018)\end{array}$ & $\begin{array}{l}\text { O estudo exclui associação entre desenvolvimento de CP e uso de liraglutida vs } \\
\text { placebo. }\end{array}$ \\
\hline 16 & Naudin S, et al. (2020) & $\begin{array}{l}\text { Aderência a um estilo de vida saudável é inversamente relacionado ao risco de } \\
\text { desenvolver CP. }\end{array}$ \\
\hline 17 & Pan X-F, et al. (2018) & Diabetes tipo 2 está associado a maior desenvolvimento de CP na China. \\
\hline 18 & Pang Y, et al. (2018) & $\begin{array}{l}\text { Tabagismo e etilismo foram fatores de risco independentes para desenvolvimento } \\
\text { de CP. Ademais menor consumo de frutas e vegetais está associado a maior risco } \\
\text { de CP. }\end{array}$ \\
\hline 19 & Park SK, et al. (2020) & $\begin{array}{l}\text { Síndrome metabólica é um fator de risco em potencial para CP. A presença de } 4 \\
\text { ou mais componentes levou a um maior risco de CP. }\end{array}$ \\
\hline 20 & $\begin{array}{l}\text { Quist AJL, et al. } \\
\text { (2018) }\end{array}$ & $\begin{array}{l}\text { O resultado sugere que a ingestão de nitrito de carne processada é um fator de } \\
\text { risco para CP. }\end{array}$ \\
\hline 21 & $\begin{array}{l}\text { Rijckers AP, et al. } \\
(2017)\end{array}$ & $\begin{array}{l}\text { Não evidenciaram aumento do risco de neoplasia de pâncreas dentro do grupo de } \\
\text { pancreatite aguda que não progrediu para cronicidade. }\end{array}$ \\
\hline 22 & $\begin{array}{l}\text { Schulpen M, et al. } \\
(2019)\end{array}$ & $\begin{array}{l}\text { Não identificaram relação entre a dieta do Mediterrâneo e o desenvolvimento de } \\
\text { câncer pancreático }\end{array}$ \\
\hline 23 & $\begin{array}{l}\text { Schulte A, et al. } \\
(2016)\end{array}$ & $\begin{array}{l}\text { A presença de história familiar positiva para câncer pancreático dobra o risco para } \\
\text { desenvolver tal neoplasia em relação ao grupo controle. }\end{array}$ \\
\hline 24 & $\begin{array}{l}\text { Setiawan VW, et al. } \\
(2019)\end{array}$ & $\begin{array}{l}\text { Diabetes recém-diagnosticado aumenta } 2,3 \text { vezes o risco de desenvolver } \\
\text { neoplasia de pâncreas. }\end{array}$ \\
\hline 25 & $\begin{array}{l}\text { Tucker MA, et al. } \\
\text { (2018) }\end{array}$ & $\begin{array}{l}\text { O único câncer que apresentou aumento do risco de desenvolvimento foi o } \\
\text { pancreático nas famílias susceptíveis a melanoma. }\end{array}$ \\
\hline 26 & Wise $\mathrm{J}(2016)$ & $\begin{array}{l}\text { Diabetes tipo } 1 \text { têm maior risco de desenvolver câncer de fígado, pâncreas, rim, } \\
\text { endométrio e ovário. }\end{array}$ \\
\hline 27 & Zheng J, et al. (2018) & $\begin{array}{l}\text { Não foi observado associação direta entre o potencial inflamatório da alimentação } \\
\text { e risco de desenvolver a entidade em questão. }\end{array}$ \\
\hline 28 & Zheng Z, et al. (2019) & $\begin{array}{l}\text { Intervenção cirúrgica precoce em pancreatite crônica pode ser fator protetor para } \\
\text { CP. }\end{array}$ \\
\hline
\end{tabular}

Fonte: Mello DMS, et al., 2021. 
O câncer pancreático apresenta uma das maiores razões de mortalidade-incidência e é responsável por aproximadamente 4\% das mortes globais por câncer (SCHULTE A, et al., 2016). 57,3\% dos casos possuem neoplasia afetando cabeça de pâncreas e 20,3\% foram diagnosticados com metástase a distância (ABDELREHIM MG, et al., 2018).

O estudo de Luo G, et al. (2019) sobre a tendência e padrão epidemiológico global concluiu que em 41 países estudados, todos apresentaram disparidades na incidência entre os sexos. No entanto, não foi possível chegar a um resultado consistente acerca do motivo da desproporção, visto que não foram considerados outros fatores que poderiam interferir nessa estatística.

O câncer pancreático apresenta incidência de câncer de pâncreas entre os povos. Huanz BZ, et al. (2019) realizaram um estudo tipo coorte multiétnico e evidenciou que os japoneses têm maior risco de desenvolver neoplasia de pâncreas, frente aos brancos europeus. Enquanto latinos apresentam a menor probabilidade de desenvolver a patologia. Porém é obscuro essa relação, já que não foi abordado os fatores de riscos ambientais pertinentes a cada região.

Dentre os cânceres pancreáticos, $90 \%$ é adenocarcinoma ductal de pâncreas exócrino e tem a maior taxa caso-mortalidade de qualquer outro câncer. Por outro lado, tumores neuroendócrinos pancreáticos (PNET) representam aproximadamente $1,3-2,8 \%$ da incidência de neoplasias pancreáticas. Embora sejam raros, os PNET possuem prognóstico ruim por apresentarem metástases à distância e serem inoperáveis em tempo do diagnóstico (ZHENG J, et al., 2018).

Poucos estudos são direcionados para PNET. Em seu estudo, Ben $Q$ et al. (2016) apontaram uma variedade de possíveis exposições relacionadas ao risco de PNETs, incluindo história de diabetes mellitus tipo 2 (DM2), consumo de álcool, tabagismo e história familiar de qualquer câncer em parentes de primeiro grau.

Embora muito se saiba da possível ligação entre fatores determinantes da dieta e do estilo de vida, níveis de folato podem ser influenciados por fatores genéticos como polimorfismo de único nucleotídeo (SNPS). Vários estudos observaram que danos ao DNA estavam intimamente ligados ao desenvolvimento de tumores, portanto como a via do folato está ligada ao reparo e síntese de DNA, postula-se que essa via tenha interferência direta no risco de neoplasia pancreática (CHITTIOBOYINA S, et al., 2018).

Chittioboyina S, et al. (2018) avaliaram a interação entre níveis de folato e o polimorfismo genético em suas vias na incidência de câncer pancreático. Nesse estudo foi constatado que, embora níveis de folato sérico não fossem estatisticamente diferentes entre os casos e controles, houve uma diferença significativa entre a distribuição genotípica de choline dehydrogenase (CHDH L78 SNP). Assim, foi identificado que sujeitos portadores do genotipo LL (nucleotídeo TT) da SNP CHDH L78 têm menor risco de desenvolver câncer de pâncreas, mesmo após ajuste de idade e sexo.

Ademais, não foram encontradas associações significantes entre risco de desenvolver câncer pancreático e outras SNPs na via do folato. Recentemente também foi apontado que o $\mathrm{CHDH}$ estimula a autofagia e consequente dano mitocondrial. Como a autofagia foi demonstrada como promotor de câncer de pâncreas, espera-se que essa mudança cause a inabilidade de estimulá-la e, por conseguinte, promova alguma proteção (CHITTIOBOYINA S, et al., 2018).

Apontou-se a relação entre telômeros e desenvolvimento de diversos cânceres, uma vez que desempenham papel importante na manutenção da integridade cromossômica. Luu HN, et al. (2019), em estudo prospectivo, ao investigarem associação entre comprimento de telômeros leucocitários, observaram possível relação entre maior comprimento de telômeros e incidência de neoplasia pancreática. No entanto, tal correlação somente foi estabelecida para adenocarcinoma pancreático.

O câncer pancreático é definido como do tipo familiar quando presente em no mínimo dois parentes de primeiro grau e este é responsável por aproximadamente $5-10 \%$ dos casos. Destes, menos de $20 \%$ são devido heranças de condições geneticamente conhecidas que predispõem ao seu desenvolvimento como: pancreatite hereditária, câncer colorretal não polipóide, neoplasias de mama e ovário hereditárias (associadas 
aos genes BRCA1 e BRCA2), síndrome melanoma múltiplo atípico familiar, síndrome Peutz-Jeghers e telangiectasia-ataxia (SCHULTE A, et al., 2016).

A identificação dos padrões de cânceres familiares pode revelar anormalidades genéticas que contribuem para o desenvolvimento dessa neoplasia. As neoplasias pancreáticas têm sido associadas a pessoas com histórico familiar de cânceres de pâncreas, mama, próstata, cólon, estômago, fígado, rins, pulmão e ovário. Entretanto, evidências em níveis populacionais são escassas (SCHULTE A, et al., 2016).

O estudo caso-controle, realizado na Austrália, não observou associação positiva entre histórico familiar de câncer de mama, ovário, pulmão e próstata e ocorrência de neoplasias pancreáticas. Todavia, observouse que a presença de história familiar positiva para câncer pancreático dobra o risco para desenvolver tal neoplasia em relação ao grupo controle (SCHULTE A, et al., 2016).

Ademais, Tucker MA, et al. (2018) acompanharam famílias suscetíveis a melanoma por quatro décadas e evidenciaram que, após melanoma, o único câncer que apresentou aumento significativo do risco de desenvolvimento nesses indivíduos foi o pancreático. Enquanto Rijkers AP, et al. (2017) perceberam que a incidência de neoplasia de pâncreas foi maior dentre os pacientes que tinham história prévia de tumores de pâncreas, vesícula, timo e mama. Além disso, mesmo após a cura dos tumores primários, o aumento da incidência de câncer pancreático nesses pacientes manteve-se maior que na população geral.

Fatores alimentares e dietéticos também possuem papel na etiopatogenia do câncer de pâncreas. A dieta pode ser um fator modulador da inflamação, a qual possui protagonismo na etiologia das neoplasias pancreáticas, uma vez que estados pró-inflamatórios são fatores de risco consolidados. Em seu estudo de coorte retrospectivo, Zheng J, et al. (2018) examinou a associação entre o potencial inflamatório da dieta e risco de câncer pancreático no ensaio clínico Prostate, Lung, Colorectal and Ovarian Cancer Screening Trial (PCLO) porém não foi observado associação direta entre o potencial inflamatório da alimentação e risco de desenvolver a entidade em questão.

O estudo retrospectivo por Quist AJL et al. (2018) investigou a associação entre câncer pancreático com o consumo de água contaminada com nitrato/nitrito em um coorte de mulheres em lowa durante o período de 1986 a 2011. Os nitritos/nitratos são ingeridos por humanos por meio da alimentação e água contaminadas e, como o nitrato é a base de muitos fertilizantes, é frequente a contaminação das fontes de água em áreas rurais. Os produtos da transformação endógena desses elementos podem ser classificados como possíveis carcinógenos humanos. São também encontrados em carnes vermelhas processadas e, em conjunto com alta concentração de ferro heme, potencializam a formação de carcinógenos.

Na análise multivariável de Quist AJL, et al. (2018) não foi descrita associação entre ingestão total de nitrato/nitrito e neoplasia de pâncreas. Entretanto, nesta análise observou-se associação positiva entre ingestão de nitrito proveniente de carnes processadas e desenvolvimento de câncer de pâncreas. Baseado em dados epidemiológicos de câncer colorretal e evidência acumulada de outros cânceres, a carne processada tem sido classificada como um carcinógeno humano. Nela estão presentes constituintes possivelmente carcinogênicos, como aminas heterocíclicas e hidrocarbonetos policíclicos aromáticos, que possuem efeito mutagênico, com estudos prévios demonstrando associação com câncer pancreático.

Gordon-Dseagu VLZ, et al. (2017) realizaram comparações entre a alta e baixa ingestão de diversos grupos alimentares e observaram que a alta ingesta de gordura resultou em aumento do risco para desenvolver neoplasia de pâncreas, enquanto o alto consumo de cálcio ofereceu um efeito protetor em relação a esse câncer.

Schulpen M, et al. (2019) realizaram uma análise de dois coortes na Alemanha e não identificaram relação entre a dieta do Mediterrâneo, baseada em alto consumo de vegetais, peixes e pouca ingestão de gordura, e o desenvolvimento de câncer pancreático. Contrapondo estudos anteriores, Abdelrehim MG, et al. (2018) não evidenciaram aumento de risco de neoplasia associado ao consumo de processados ou baixa ingestão de peixes.

O estudo de intervenção realizado com 111348 mulheres no grupo de intervenção e 17652 no grupo de intervenção placebo, por Jiao L, et al. (2018), ratificou que a ingestão menor de gordura e maior de vegetais, 
frutas e grãos não tem impacto significativo no aumento ou redução do risco de câncer de pâncreas. Porém, tal estudo observou uma redução do risco em mulheres obesas que reduziram o grau de obesidade, mostrando uma relação entre obesidade e incidência da neoplasia em questão.

A obesidade é um dos fatores de risco para o desenvolvimento de doenças crônicas e tumorais. Sua concomitância com outras comorbidades potencializam a probabilidade de desenvolver diversos cânceres. Sabe-se que a obesidade promove um estado pró-inflamatório corporal, o que poderia sustentar esses achados. Naudin S, et al. (2020) realizaram um estudo prospectivo para investigar a relação entre fatores de estilo de vida e câncer pancreático e concluiu que, além de hábitos não saudáveis, a combinação com obesidade aumentou a incidência de câncer em adultos.

Corroborando com tais achados, Koyanagi YN, et al. (2018) fizeram uma meta-análise com inclusão de coortes japonesas para avaliar a relação entre o índice de massa corpórea (IMC) e câncer pancreático, e evidenciaram uma associação significativamente positiva entre obesidade e risco de neoplasia de pâncreas, principalmente em homens.

A concordância entre diabetes e diferentes tipos de câncer é pesquisada em diversas populações e desenhos de estudos. Meta-análises sugerem robusta relação entre diabetes mellitus tipo 2 e desenvolvimento de câncer de mama, colangiocarcinoma intra-hepático, câncer colorretal e endometrial. Pan $\mathrm{X}-\mathrm{F}$, et al. (2018) estudaram 500 mil chineses em estudo prospectivo cercando a associação entre DM2 e risco de câncer, concluindo que o DM2 não somente aumentou o risco geral de câncer como aumentou o risco de neoplasia pancreática em $86 \%$.

O estudo de Setiawan VW, et al. 2019, observou que diabetes recém-diagnosticado aumenta 2,3 vezes o risco de desenvolver neoplasia de pâncreas em contraste com a diabetes de longa data, demonstrando uma possível relação causa-consequência entre as duas entidades. Embora muitos estudos abordem apenas a DM2, Wise $\mathrm{J}$ (2016) estudou o risco geral de desenvolver câncer relacionado com diabetes mellitus tipo 1 (DM1) e concluiu que DM1 têm maior risco de desenvolver câncer de fígado, pâncreas, rim, endométrio e ovário.

Diabetes e obesidade são fatores de risco para diversos tipos de câncer e mortalidade por câncer e, por isso, medicamentos podem modificar o risco de tais eventos. O Estudo duplo cego randomizado realizado por Nauck MA, et al. (2018) analisou o risco de neoplasias associado a liraglutida versus placebo. Neoplasias de pâncreas e tireoide foram confirmadas em $10,1 \%$ dos usuários de liraglutida, em comparação à $9 \%$ dos pacientes que receberam placebo.

Algumas drogas são notadamente responsáveis por alterar a patogênese tumoral. Sabe-se que a digoxina é um medicamento que afeta diversas vias envolvidas na tumorigênese. Ela é utilizada para tratamento de disfunções cardíacas e, nos últimos anos, muito se observa sobre seus os efeitos antineoplásicos, com estudos demonstrando que seu uso está relacionado à significante redução da incidência de cânceres (BOURSI B, et al. 2021).

Boursi B, et al. (2021) identificaram, em estudo caso-controle, que a digoxina não apresenta efeito protetor neste caso, mas que a relação inversa se mostrou verdadeira. Neste estudo, a digoxina aumentou a incidência de neoplasia de pâncreas em $41 \%$ quando comparado aos controles, com manutenção do risco mesmo após um ano de suspensão da droga.

Outra droga elencada como possível fator de risco para neoplasia de pâncreas foi o ópio, que de acordo com Moossavi S, et al. (2018), há uma relação positiva entre incidência de câncer pancreático e consumo de tal droga. Por ser um resultado inédito, não se sabe ainda se esse risco foi um achado estaticamente coerente e nem seu mecanismo. Necessita-se de estudos adicionais que verifiquem se o risco também está presente com o uso de medicamentos derivados, como os analgésicos opióides.

De acordo com Quist AJL, et al. (2018) apenas o tabagismo e a pancreatite crônica são fatores de risco bem estabelecidos para neoplasia pancreática. A pancreatite crônica é uma condição inflamatória crônica. Sabe-se que condições inflamatórias constituem predisposição para cânceres de órgão específico. Portanto, 
é possível associar pancreatite crônica a um maior risco para o desenvolvimento de neoplasia pancreática, evidenciado por vários estudos. A pancreatite crônica pode ser causada por diversos fatores, dentre etilismo, hiperlipidemia, hereditária e autoimune.

Hao L, et al. (2017) evidenciaram que a taxa de incidência de neoplasia pancreática é aumentada nos portadores de pancreatite crônica, sobretudo quando associado ao tabagismo e idade avançada. $O$ estudo retrospectivo de Zheng Z, et al. (2019) corrobora com o anterior e acrescenta que quanto mais grave e avançada a pancreatite, maior a incidência de câncer de pâncreas.

Não obstante os estudos foquem na pancreatite crônica, Rijkers AP, et al. (2017) pesquisaram a incidência de neoplasia de pâncreas em pacientes com episódio primário de pancreatite aguda e não evidenciaram aumento do risco de neoplasia de pâncreas dentro do grupo que não progrediu para cronicidade.

É bem conhecido que o etilismo e tabagismo são responsáveis por aumentar a incidência de neoplasias em geral, como também estão associados com diagnósticos de tumores mais avançados. Tal fato se justifica por mecanismo de lesão direta ao DNA celular e vias de reparo, ativação de cascatas de inflamação ou deficiência de nutrientes secundários (BEN Q, et al. 2016).

Vários estudos tiveram como objetivo associar esses hábitos ao desenvolvimento de neoplasias pancreáticas. Na meta-análise de dez estudos tipo coorte, Koyanagi YN, et al. (2019) observaram que tabagistas ativos tiveram maior risco de desenvolver neoplasia de pâncreas em ambos os sexos, frente ao grupo que nunca foi tabagista. Ademais, foi encontrado que mulheres ex-tabagistas ou tabagistas de pequena monta (<20 maços-ano) tiveram risco aumentado de desenvolver câncer pancreático. Observou-se uma redução do risco de câncer de pâncreas em homens após cinco anos de cessação do tabagismo.

Corroborando com achados prévios, Hao L, et al. (2017) observaram que o tabagismo aumentou significativamente a incidência de neoplasia pancreática, especialmente dentre grandes tabagistas (>60 maços-ano). No estudo prospectivo realizado com 500 mil pacientes feito por Pang Y, et al. (2018), o tabagismo e etilismo foram fatores de risco independentes para o desenvolvimento de neoplasia pancreática. Todavia, em análise multivariada, Ben Q, et al. (2016) não observaram associação entre o etilismo e um maior risco de desenvolver neoplasia de pâncreas, com exceção à PNET.

\section{CONSIDERAÇÕES FINAIS}

A neoplasia de pâncreas, embora possua baixa prevalência, apresenta uma elevada taxa de mortalidade. Possui etiologia multifatorial, que compreende aspectos ambientais, inflamatórios e genéticos e possui como fatores de risco robustos a presença de pancreatite crônica, tabagismo e história prévia familiar de melanoma ou neoplasia pancreática. Todavia, outros fatores como a dieta e alguns genes vêm demonstrando papel importante na incidência dessa patologia. $O$ câncer pancreático possui terapêutica limitada e necessita de diagnóstico em estágios iniciais para o sucesso do tratamento. Diante das evidências, conclui-se, portanto, que o reconhecimento dos fatores de risco para a neoplasia de pâncreas é de extrema importância para sua prevenção, diagnóstico precoce e sucesso terapêutico, a fim de possibilitar melhora do perfil epidemiológico atual.

\section{REFERÊNCIAS}

1. ABDELREHIM MG, et al. Dietary Factors Associated with Pancreatic Cancer Risk in Minia, Egypt: Principal Component Analysis. Asian Pac J Cancer Prev, 2018; 19(2):449-455

2. BEN Q, et al. Risk factor for sporadic pancreatic neuroendocrine tumors: case control study. Scientific Reports, 2016; 6:36073.

3. BOURSI B, et al. Digoxin use is associated with pancreatic cancer risk but does not affect survival. Cancer Causes Control, 2021; 32(1):41-46.

4. CHITTIBOYINA S, et al. The role of the folate pathway in pancreatic cancer risk. PLoS One, 2018; 13(2):e0193298.

5. GORDON-DSEAGU VLZ, et al. A Cohort Study of Adolescent and Midlife Diet and Pancreatic Cancer Risk in the NIHAARP Diet and Health Study. Am J Epidemiol, 2017; 186(3):305-317. 
6. HAO L, et al. Incidence of and risk factors for pancreatic cancer in chronic pancreatitis: A cohort of 1656 patients. Dig Liver Dis, 2017; 49(11):1249-1256.

7. HUANG BZ, et al. Interethnic differences in pancreatic cancer incidence and risk factors: The Multiethnic Cohort. Cancer Med, 2019; 8(7):3592-3603.

8. JIAO L, et al. Low-fat Dietary Pattern and Pancreatic Cancer Risk in the Women's Health Initiative Dietary Modification Randomized Controlled Trial. J Natl Cancer Inst, 2018; 110(1):49-56.

9. KAMATH GR, et al. Risk of Primary Neuroendocrine Pancreatic Tumor After a First Primary Cancer: A US PopulationBased Study. Pancreas, 2019; 48(2):161-168.

10. KOYANAGI YN, et al. Body-Mass Index and Pancreatic Cancer Incidence: A Pooled Analysis of Nine PopulationBased Cohort Studies with More Than 340,000 Japanese Subjects. J Epidemiol, 2018; 28(5):245-252.

11. KOYANAGI YN, et al. Smoking and Pancreatic Cancer Incidence: A Pooled Analysis of 10 Population-Based Cohort Studies in Japan. Cancer Epidemiol Biomarkers Prev, 2019; 28(8):1370-1378.

12. LUO G, et al. Global Patterns and Trends in Pancreatic Cancer Incidence: Age, Period, and Birth Cohort Analysis. Pancreas, 2019; 48(2):199-208.

13. LUU HN, et al. Association between leukocyte telomere length and the risk of pancreatic cancer: Findings from a prospective study. PLoS One, 2019; 14(8):e0221697.

14. MIZRAHI JD, et al. Pancreatic cancer. Lancet, 2020; 395(10242): 2008-2020.

15. MOOSSAVI S, et al. Opium Use and Risk of Pancreatic Cancer: A Prospective Cohort Study. Cancer Epidemiol Biomarkers Prev, 2018; 27(3):268-273.

16. NAUCK MA, et al. Neoplasms Reported with Liraglutide or Placebo in People with Type 2 Diabetes: Results From the LEADER Randomized Trial. Diabetes Care, 2018; 41(8):1663-1671.

17. NAUDIN S, et al. Healthy lifestyle and the risk of pancreatic cancer in the EPIC study. Eur J Epidemiol, 2020;35(10):975-986.

18. PAN X-F, et al. Type 2 Diabetes and Risk of Incident Cancer in China: A Prospective Study Among 0.5 Million Chinese Adults. Am J Epidemiol, 2018; 187(7):1380-1391.

19. PANG Y, et al. Smoking, alcohol, and diet in relation to risk of pancreatic cancer in China: a prospective study of 0.5 million people. Cancer Med, 2018; 7(1):229-239.

20. PARK SK, et al. Metabolic syndrome, metabolic components, and their relation to the risk of pancreatic cancer. Cancer, 2020; 126(9):1979-1986.

21. PEREIRA SP, et al. Early detection of pancreatic cancer. Lancet Gastroenterol Hepatol, 2020; 5(7): 698-710.

22. QUIST AJL, et al. Ingested nitrate and nitrite, disinfection by-products, and pancreatic cancer risk in postmenopausal women. Int J Cancer, 2018; 142(2):251-261.

23. RIJKERS AP, et al. Risk of Pancreatic Cancer After a Primary Episode of Acute Pancreatitis. Pancreas, 2017; 46(8):1018-1022.

24. SCHULPEN M, et al. Mediterranean diet adherence and risk of pancreatic cancer: A pooled analysis of two Dutch cohorts. Int J Cancer, 2019; 144(7):1550-1560.

25. SCHULTE A, et al. Association between family cancer history and risk of pancreatic cancer. Cancer Epidemiology, 2016; 45:145-150.

26. SETIAWAN VW, et al. Pancreatic Cancer Following Incident Diabetes in African Americans and Latinos: The Multiethnic Cohort. J Natl Cancer Inst, 2019; 111(1):27-33.

27. TUCKER MA, et al. Risks of Melanoma and Other Cancers in Melanoma-Prone Families over 4 Decades. J Invest Dermatol, 2018; 138(7):1620-1626.

28. WISE J. Type 1 diabetes is associated with increased risk of several cancers. BMJ, 2016; 352:i1210.

29. ZHENG J, et al. Inflammatory potential of diet and risk of pancreatic cancer in the Prostate, Lung, Colorectal and Ovarian (PLCO) Cancer Screening Trial. Int J Cancer, 2018; 142(12):2461-2470.

30. ZHENG Z, et al. Risk of pancreatic cancer in patients undergoing surgery for chronic pancreatitis. BMC Surg, 2019; 19(1):83. 